\title{
DUPLICATION OF THE STOMACH
}

\author{
BY \\ W. H. GALLOWAY, ANNE M. SUTHERLAND and A. WYNN WILLIAMS \\ From the Departments of Child Health and Pathology, University of Aberdeen
}

(RECEIVED FOR PUBLICATION APRIL 26, 1956)

Duplications of the alimentary tract, and of the stomach in particular, are seen very rarely and for this reason alone they may escape diagnosis. It is, therefore, considered instructive to describe a case of gastric duplication treated successfully by resection.

\section{Case Report}

A female infant aged 4 weeks was admitted to the City Hospital, Aberdeen, on March 31, 1955. Ten days previously she had an episode of vomiting, and on the day of admission she passed a green, slimy stool. At birth she weighed $9 \mathrm{lb}$. $4 \mathrm{oz}$. and delivery was spontaneous at full term. She was fed on breast milk for two weeks after birth but the supply became insufficient. Before admission she was receiving $5 \mathrm{oz}$. of a mixture of cow's milk and water four-hourly five times daily.

Her brother, aged 5, and her mother were well, but the father was in hospital with acute nephritis.

On examination the baby was small but not dehydrated. Her weight $(8 \mathrm{lb} .10 \mathrm{oz}$.) indicated retarded growth. A smooth swelling, $1 \times 2$ in. in area, was visible in the left hypochondrium; it was firm and apparently fixed but it could be partially surrounded by one's fingers. When the baby was fed, however, the swelling became firmer and moved downwards and inwards. No peristalsis was seen. A mouthful of feed was possetted but no true vomiting occurred.

Four days after admission a barium meal was given but there were no diagnostic radiological findings. The abdominal mass remained unchanged. However, the baby fed well and gained weight until April 12 when she developed symptoms of gastro-enteritis due to crossinfection with $E$. coli O111B4. On one occasion during this illness frank blood was observed in the stool. Despite antibiotic (tetracycline) therapy cultures of stool remained positive. The baby gradually regained her health, however, her appetite returned and she gained in weight, but the abdominal swelling remained unchanged.

On July 1 radiological examination was repeated. No abnormality was detected after a barium meal until the baby sucked from an empty bottle and thus distended the stomach with air. The tumour was then seen protruding into the stomach and appeared to be attached to the middle part of the greater curvature (Fig. 1). Laparotomy was advised and the baby was therefore transferred to the Royal Hospital for Sick Children, Aberdeen, for surgical treatment.

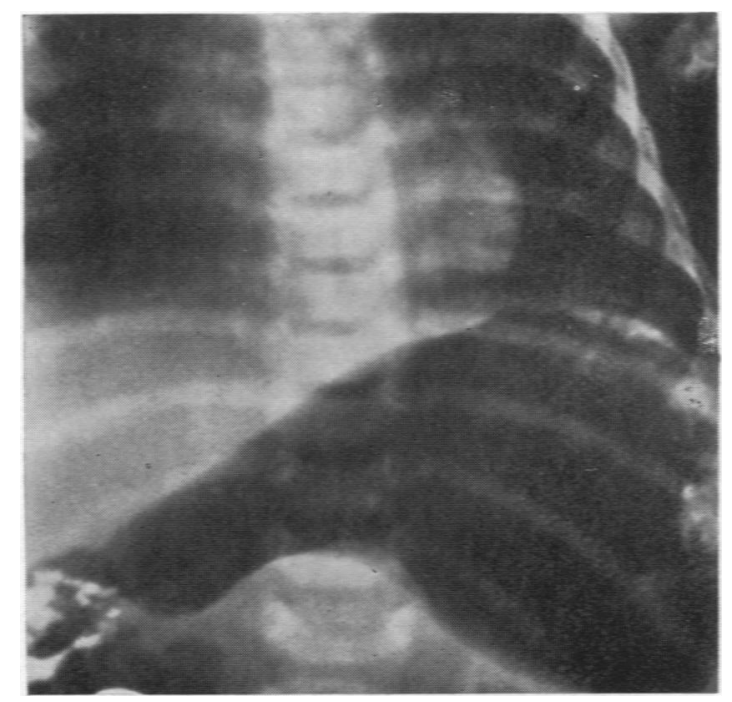

FIG. 1.-Radiological appearances of the stomach (antero-posterior view) after a barium meal. The duplication protrudes into the lumen of the stomach at the greater curve.

Operation. Under general anaesthesia (gas, oxygen, ether) Mr. S. G. Davidson performed, a laparotomy through a right paramedian incision. A smooth, oval cystic mass, approximately $2 \times 1 \times 1$ in., was attached midway along the greater curve of the stomach and was also bound by fibrous adhesions to the transverse colon and pancreas. Much of the wall appzared to blend with that of the stomach, while at one point it was firmly adherent to the colon, the app zarances suggesting a small, partially-healed inflammatory and ulcerated focus. After dissection from the colon, the mass was removed together with a fringe of stomach wall, $\frac{1}{2}$ in. wide, and a small piece of pancreas. No communication was observed batwzen the cavity of the cystic lesion and the stomach lumen. After the resection the stomach wall was sutured and an aspiration tube manipulated into the duodenum. 
Pathological Findings. The specimen consisted of a cystic structure shaped like a kettledrum (Fig. 2) and measuring $5 \times 3 \times 3 \mathrm{~cm}$. It had a thick muscular wall which merged into the muscle layer of the attached ovalshaped portion of true stomach and it was covered exteriorly by peritoneum. At one point on its lower convex border there was a small granulomatous nodule surrounding a minute orifice. Some $2 \mathrm{ml}$. of slightly mucinous but otherwise watery fluid was 'milked' through this hole by compression. The fluid had a $p H$ of 4.0 and contained pepsin but no free hydrochloric acid. When the specimen was bisected a cavity was seen, approximately $3 \mathrm{~cm}$. in diameter lined by pink, healthy-looking mucous membrane partially folded into small rugae (Fig. 3). There was no communication between the cavity and the overlying stomach.

Microscopic examination of the cystic structure showed that its mucous lining was like that of normal gastric mucosa (Fig. 4), with chief and parietal cells easily identifiable. There were no active erosions or ulcers, but the appearances of the mucosa around the small orifice on the distal border, where there had been adhesion to the transverse colon, were suggestive of healed erosion. There was a well-defined muscularis mucosae beneath the mucous membrane and external to this were a submucous layer of connective tissue and then a thick muscle coat, most of which was covered by peritoneum. In many parts of the cavity wall two muscle coats, an inner circular and an outer longitudinal, were distinguishable; these blended with the muscle coat of the true stomach. thus accounting for the surgical impossibility of removing the cyst by dissection. Auerbach's and Meissner's nerve plexuses appeared to be normal.

The appearances thus conformed to those of so-called 'duplication of stomach'.

Progress. The condition of the baby after operation was uneventful except for some reluctance to take feeds. She was discharged home August 4, when she weighed $11 \mathrm{lb} .12 \mathrm{oz}$. On January 4, 1956, there was still a little difficulty in feeding but the weight was $17 \mathrm{lb} .4 \mathrm{oz}$. A further radiological examination revealed no abnormality of the vertebrae, particularly in the cervical and upper dorsal region, but an opacity was seen in the right lung field at the level of the hilum (Fig. 5). It did not pulsate and it caused no oesophageal displacement. Moreover, no calcification was seen in it. The significance of this abnormality is uncertain but it may be a mediastinal cyst of foregut origin. No surgical interference is at present contemplated, but the infant is being kept under close observation.

\section{Discussion}

The baby was admitted to hospital because of green, slimy stools and failure to thrive, not because of an abdominal tumour. When the latter was observed clinically it was thought to be either a double stomach or a cyst. The correct diagnosis of duplication of stomach was suggested by radio- logical examination after a barium meal when the abnormality was seen to be outside the stomach but could yet be invaginated into it.

Duplications are 'spherical or elongated hollow structures which possess a coat of smooth muscle, which are lined by a mucous membrane, and which are intimately attached to some portion of the alimentary tube' (Ladd and Gross, 1941). They are more common in relation to small intestine than to any other part of the alimentary tract, and they are usually observed in childhood but may be found at any age. The lining is alimentary in type but does not necessarily correspond to that at the level at which the duplication is found, e.g., a rectal cyst may have a partial lining of gastric mucosa. In some specimens there are two or even three types of alimentary mucosa. In Ladd and Gross's series of 18 cases of duplication the sites were: base of tongue, in one patient; oesophagus, in three; stomach, in one; duodenum, in one; jejunum, in two; ileum, in seven; caecum, in one; sigmoid, in one; rectum, in one. Their case involving the stomach was in a 6-year-old girl; the lesion was on the greater curve and posterior surface and was almost as large as the stomach.

Duplications may or may not communicate with the lumen of the adjacent hollow viscus. In the case under discussion there appeared to have been ulceration from the duplication into the transverse colon; this, we presume, was a possible cause of melaena.

Signs and symptoms of duplication arise most commonly from mechanical obstruction to part of the normal alimentary tube. Repeated haemorrhage, associated with peptic ulceration, has also been described. The radiological findings are very helpful in diagnosis, while at laparotomy the intimate union of the muscle coat of the duplication with that of the adjacent alimentary tract is important in the recognition of the condition. Mesenteric cysts which may be confused with abdominal duplications can usually be dissected free and are, moreover, thin-walled. In our patient the movement of the abdominal swelling, observed clinically, is attributable to the firm attachment of the duplication to the greater curve of stomach: as the stomach became distended with food, the swelling moved downwards and inwards towards the midline.

Several theories have been advanced to explain the formation of duplications. The most popular is the 'sequestration theory', viz., groups of cells from the primordial intestinal tube become pinched off. In this connexion it is of interest that Lewis and Thyng (1907) frequently found diverticula in various portions of foetal alimentary tract in pigs. 


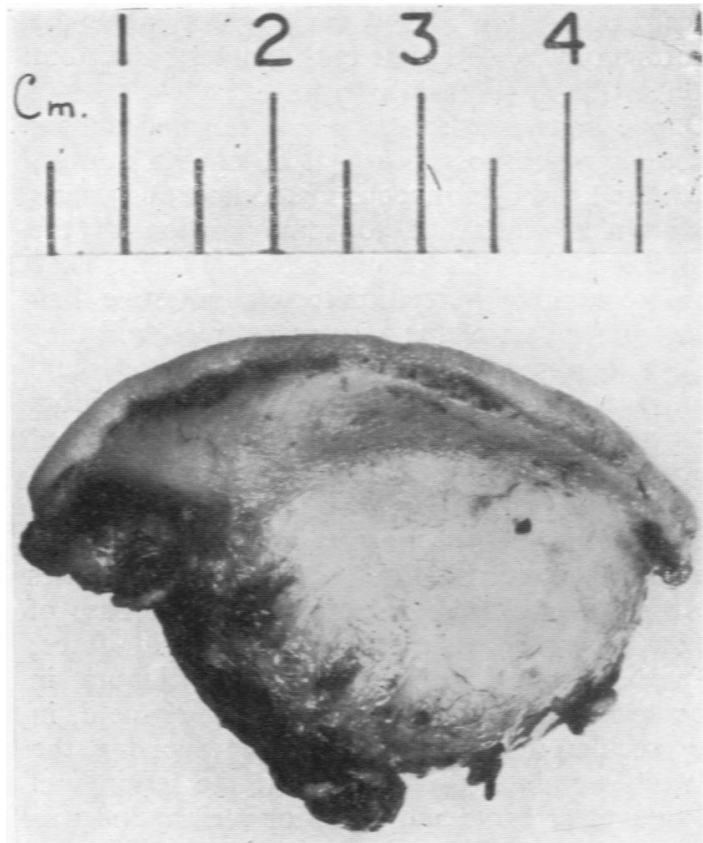

FIG. 2.-Lateral view of the resected specimen. The upper part is covered by a part of the normal stomach wall.

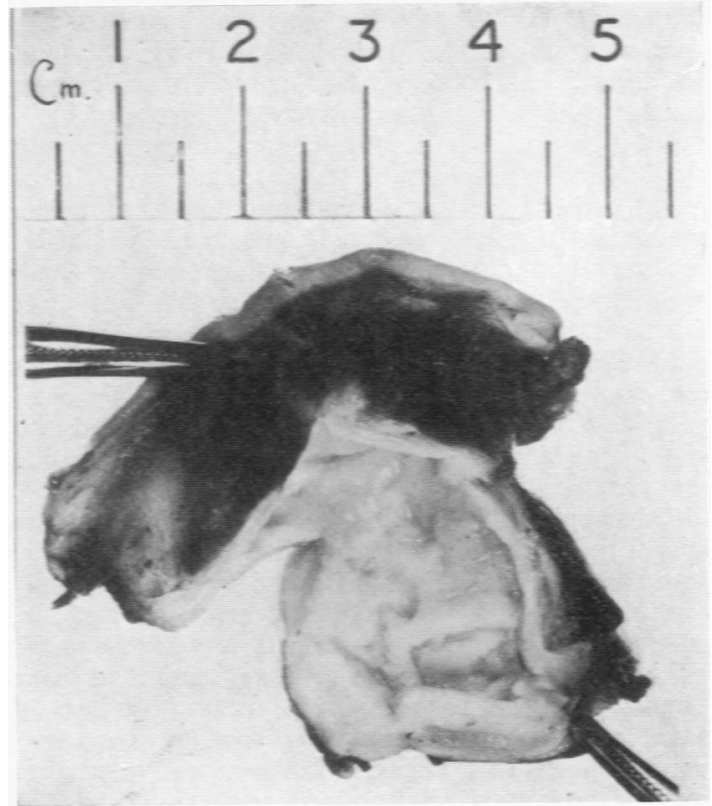

Frg. 3.-The specimen after bisection showing its rugous mucous lining.

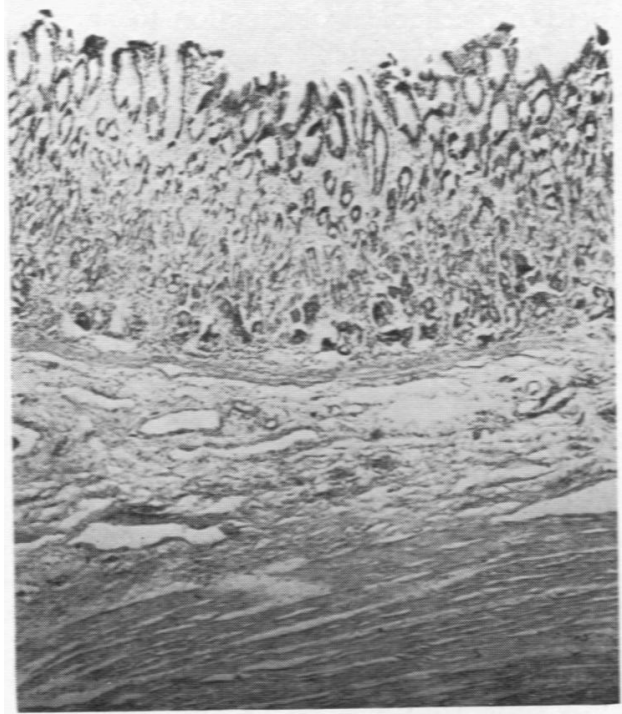

Fig. 4.-Section of the wall of the duplication showing a mucous membrane like that of normal body of stomach. Hacmatoxylin and eosin, $\times 40$.

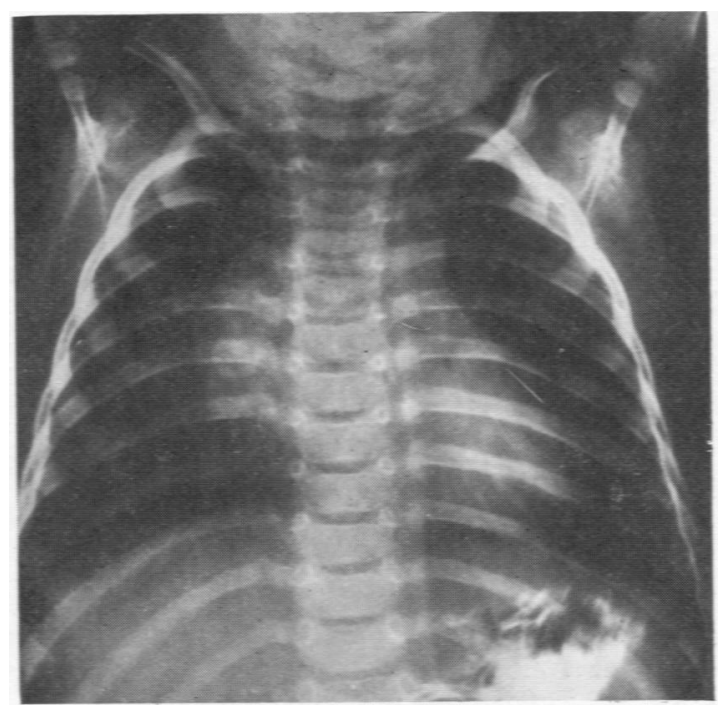

Fig. 5.-Radiological appearances of the thorax (antero-posterior view) showing the mediastinal opacity. 
rabbits, cats, sheep and man. These were seen most often in the ileum but were unrelated to a Meckel's diverticulum and usually regressed.

We have mentioned the radiological finding of an opacity in the chest and have suggested the possibility of it being due to a mediastinal cyst of foregut origin. Fallon, Gordon and Lendrum (1954) have given an excellent account of such cysts which are commonly associated with vertebral anomalies and sometimes with mesenteric cysts and duplications. They emphasize that the association of such mediastinal cysts with anterior spina bifida has not been adequately appreciated and they regard such cysts as the final result of an abnormal extension of the lumen of the foregut produced on its posterior aspect by incomplete separation of the notochord. Consequent interference with anterior fusion of the vertebrae brings about the vertebral anomalies. Later in embryonic development the cyst tends to move caudally by growth of the intrathoracic viscera.

\section{Summary}

A case of duplication of the stomach is described and points of diagnostic value are indicated.

We are indebted to Mr. S. G. Davidson, Dr. A. M. Stewart and Professor J. S. Young for their help and advice.

\section{REFERENCES}

Fallon, M., Gordon, A. R. G. and Lendrum, A. C. (1954). Brit. J. Surg., 41.520.

Ladd, W. E. and Gross, R. E. (1941). Abdominal Surgery of Infancy and Childhood, p. 83. Philadelphia and London.

Lewis, F. T. and Thyng, F. W. (1907). Amer. J. Anat., 7, 505. 